\title{
Computer workstations: Explorations in human and machine cognition
}

\author{
ROY LACHMAN \\ University of Houston, Houston, Texas
}

\begin{abstract}
Professional and scientific work will increasingly be conducted at computer workstations. An expert-system adaptive interface is one method for supporting learning and raising productivity of workstation activities. A prototype application for an adaptive interface is described in terms of machine intelligence, needs, design philosophy, and cognitive research. The intelligent interface prototype includes the operator's psychometric profile, prior performance history, and a current keystroke performance stack within a conventional expert system. Productions in the form of conditionals between the operator's keystrokes and three types of commands to the underly. ing software are described and illustrated. Commands entered, syntax errors, excessive repetition of keystrokes, and skill level are used to detect the user's intentions and accordingly modify the display and command structure of the underlying software. Determining human intentions to produce machine adaptation to individual users is not a trivial task. Efforts to develop a powerful and general adaptive interface pose significant problems, and success cannot be assured without intensive cognitive research.
\end{abstract}

Integrated computer workstations are now under development at major U.S. corporations and at several government agencies. A computer workstation typically consists of a desk-top computer that stands alone or is linked to a network. The computer generally supports conventional software services such as word processing, spreadsheets, and other programming or application software. An integrated workstation provides, in addition to standard services, the specialized software that supports the professional activities of physicians, lawyers, astronauts, engineers, research scientists, or other professionals. In a factory or refinery, an integrated workstation permits a manager to monitor real-time manufacturing processes that are computer-based or equipped with sensors, and on the same equipment to access personnel and financial databases, decision support systems and any other available software. An integrated workstation aboard a spacecraft would, in addition, support command and control activities, repairs, and tasks associated with habitation.

In the 1990s, an increasing number of professionals will practice many aspects of their crafts at integrated workstations. Proprietary as well as open research and development is underway to support this trend. For example, Luchier, Matheson, Butter, and Reynolds (1988) describe the strategic plan of the Johns Hopkins Medical Institutions for a medical knowledge workstation and a clinical applications workstation. The most ambitious program, in this regard, is the Multipurpose Applications Console that will be used by the crew of U.S. Space Station Freedom. Scientific research as well as standard operations

Correspondence may be addressed to Roy Lachman, Department of Psychology, University of Houston, Houston, TX 77204-5341. aboard the space station will be conducted at the workstation interface. Crews will operate and maintain equipment of unprecedented technological sophistication and complexity. Simultaneous monitoring of multiple systems, documentation access, data management, and command and control will be routinely conducted at integrated workstation consoles. Significant components of space station design now incorporate machine intelligence in the form of expert systems, robotics, and advanced automation. One aspect of machine intelligence in the Space Station Program Requirements should be of particular interest to cognitive psychologists. The requirements call for standard computer services with knowledge-based components and for an interface with the capacity to adapt to characteristics of individual crew members.

In this paper, I will explore approaches to designing intelligence in the computer-human interface in the form of knowledge-based technologies. I will initially describe the effort that is being made to conceptualize an approach to this problem, formulate a design philosophy, and develop a prototype. I will also consider the relatively slow pace of research on adaptive systems and its significance. Cognitive psychology provides one major orientation for dealing with the problem. However, the usual distinctions between basic and applied science are misleading in the domain of human and machine cognition. The controversy regarding machine intelligence (i.e., the prospect for computer implementation of high-level, human-like capacities) is not subject to empirical verification or falsification or to resolution by formal proof. Any computer program that establishes some aspect of intelligence in a machine, by whatever means, is an existent proof that adds to the ever growing set of intelligent capacities of computers. This may be the only form of verification possible. In this sense, technology is a key contributor to a 
basic science of comparative machine cognition-perhaps the most important contributor.

\section{Needs}

However one may wish to define intelligence, adaptation must be a central attribute. Adaptive capacities for workstation software would raise overall productivity and provide special support for novice or infrequent users of a particular application. The need is particularly acute for adaptive capacities in the integrated workstations of manned spacecraft. The logistics and the cost of habitation aboard the space station, for example, will necessitate a minimum size of crew. Each crew member, therefore, will be required to do tasks outside of a particular field of specialization and will be assigned multiple responsibilities. Crew skill levels will vary with frequency of performance of particular tasks. The interface for a computer-based activity that is most suitable for peak skills can be highly unsuitable when that skill is diminished. Extended habitation in space requires that computers adapt to fixed and variable human characteristics. Intelligent machines, with knowledge-based control of the computer-human interface, are important for the effective deployment of advanced computer-human interactive technologies as well as for raising productivity in computer-based tasks that are now commonplace.

A number of cognitive science problems are related to developing an intelligent interface that adapts its displays and command structure to each individual system user. Psychometric and performance data for an individual are one source of rules or other forms of representation for the knowledge base of an expert system. This knowledgebased system is responsible for adapting the control structure and command dialogue of workstation services to the fixed characteristics and current performance level of the user. Individual capacitites measured previously and performance collected continuously at the operator-system interface have the potential for interpreting human performance, competence levels, errors, or other unsuitable performance patterns. Information obtained unobtrusively from embedded performance measures or directly from queries to the human operator serve as the input to expertsystem software modules. Elements of this information are the antecedents to the firing of rules in a productionsystem architecture. An expert-system workstation interface possesses the potential for reducing errors and significantly enhancing human performance and productivity on the space station and here on earth. However, the effort to develop a powerful adaptive interface poses significant problems, and success in the near future is not assured.

\section{Design Philosophy}

There is always a design philosophy involved in the design and development of any engineering artifact, including software systems. If not explicit, this design philosophy is implicit. It is useful to articulate such a philosophy, so that inadequacies can be recognized and corrected before changes become too costly. The design philosophy behind the present effort to produce computer adaptation is consistent with carefully developed approaches such as that of Rouse, Geddes, and Curry (198788 ); it may be summarized as follows:

- Develop operator-centered design; leave the crew or other professionals in charge.

- Overcome human sensory and cognitive limitations.

- Amplify existing human abilities.

- Automate where human performance is below an acceptable level.

- Sophistication of support for each application can vary from simple methods to large scale knowledge-based systems.

- Develop support systems incrementally within a range of the application subsystems.

- Map expert-system technology onto the problem.

The major justification for an operator-centered approach stems from the impossibility of ruling out the need for human intelligence to solve problems. Unanticipated events are inevitable; only human intelligence can deal with unanticipated events. Automated systems including robotics and expert systems can only deal with events that have been anticipated, and then only when plans have been specifically enacted to deal with these events. There are numerous examples of the failure of designers to anticipate potentially catastrophic events (Davis, 1989). An instructive example is the false warning of an impending Soviet nuclear attack in 1960, which was due to unanticipated radar reflections from a moonrise. The system managers had information about many things that could readily be entered into a knowledge base, such as "new systems tend to have bugs" and "any system may fail." However, they also knew that the Russian leader Khrushchev was in New York. The latter represents a class of information available only to a system that reads a newspaper each day and possesses an unrestricted sense of relevance and an unbounded inferential capacity; these are not likely to be qualities of computers at any time soon. An appropriate philosophy, therefore, must put human beings at the center of decision making and problem solving yet provide computer aids that can adapt to the changing limits of human skills. Expert systems are the technology of choice for an adaptive interface because of their power and flexibility and their capacity to capture the heuristics of highly skilled human performance.

\section{Research in Cognitive Psychology}

It is axiomatic in theories of intelligence that adaptability is essential to intelligent behavior. The mechanisms of adaptation must likewise be central to the concept of machine intelligence. Understanding the processes by which adaptability is constructed with a knowledge-based system requires more than artificial intelligence system building; basic research in cognitive psychology is also essential. Research to isolate the heuristics that facilitate machine adaptation to the human user's fixed and variable capacities requires combining research in artificial 
intelligence and psychology. The study of general human expertise and skilled performance in selected workstation tasks is one precondition for designing adaptive programs.

Developing an adaptive interface requires inquiry into a machine's ability to recognize user and task properties. In order to effectively guide a person through a complicated task, a machine could copy the behavior of a human advisor or tutor as a first approximation. The machine, therefore, must possess coherent knowledge of the person and the task, and it must be capable of making the correct interpretation of relevant response sequences. The expert-system database must possess detailed information about any current stage of the task and the user's performance. At certain machire-state operator-input cycles, it is essential to determine the user's intentions, at least partially, for effective machine intervention. The computational system can be designed to provide more realistic help and improved guidance if it possesses rules and facts dealing with user characteristics related to performance, such as general task knowledge, aptitude, and motivation.

Precise formulations of the user's task-state knowledge and intentions relative to the current machine state (i.e., what the user is trying to do at a given moment) is one essential contribution of cognitive psychological research. Machine-detectable user information from keystrokes is currently the primary basis for "firing" rules that produce the adaptation of a workstation's display and command structure. It is important to have the capability of detecting increasing operator expertise. Anderson, Farrell, and Sauers (1984) have shown that the development of expertise entails a shift from the use of declarative knowledge to the development of problem-specific productions that are subsequently used in new situations. The acquisition of productions can be detected by a decrease in latency and recurring keystroke patterns. Automaticity, increases in quantity of knowledge, schemadriven problem solving, and changes in chunking panterns all indicate growth of expertise (Chi, Glaser, \& Reese, 1982). Several of these indices are detectable through changes in keystroke patterns and response latencies to specific machine states. Changes in an operator's level of expertise produces patterns that change the command and display structure of a rule-driven interface.

\section{Data Management Service "Simulation"}

Workstation hardware and software components in NASA's current Space Station specifications (National Aeronautics and Space Administration, 1989) are to be designed for modularity. The software will contain a number of modules for crew operations support. These will provide, among many other functions, data management services or DMS. DMS will support a full spectrum of computer services, including document retrieval, word processing, and database access. Word processing is the DMS function used as an example in the present study. The text editor is an extension of software previously developed to enhance comprehension of technical text (Lach- man, 1989b). The software that was developed for this study is not really a simulation, but a functional word processor with a variable or adaptable command set. The virtue of the capacity to reduce the command set is that it limits the number of legal responses and simplifies the task of predicting the user's intentions from his or her keystrokes. Figure 1 shows one of several help screens with a limited command set. The following rule produces that help screen:

RULE 4

IF RESPONSE(last) $=$ ALT.H

AND IF LEARNING.LEVEL $=2$

THEN PRINT HELPSCREEN. 2

The rule states that if the operator enters the command sequence "Alt" " $\mathrm{H}$ ", and if the operator is at Learning Level 2 ( 1 is lowest), then the system will display the help screen shown in Figure 1. The value of "learning level" must be determined empirically, since there is no extant cognitive theory that can precisely inform this process. Error levels, latency of command entry, and number of hours of system use are some of the variables that can be used to autornatically set the user's current level of expertise. This value and other parameters are stored in a file and are entered into the expert-system database when a particular user logs on.

\section{The Expert System Adaptive Interface}

The core idea of an adaptive interface is to reverse the usual roles of human and computer and to equip the computer with mechanisms to learn about the user and adapt accordingly. Several approaches to human performance, task characteristics, and adaptation methodology have been proposed in the computer-human interface literature. These approaches include statistical analyses of performance, the GOMS model (Card, Moran, \& Newell, 1983), generative grammar (Hoppe, 1988; Payne \& Green, 1986), production systems (Polson \& Kieras, 1985), and hybrid formalisms (Tyler, 1988). The first approach, statistical analysis of keystroke command selection, is by itself insufficient for driving an autoadapting system, but it can be part of a more effective design that includes an expert-system driver for the workstation task interfaces. A generative grammar is formally equivalent to a production system (cf. Lachman, 1989a), but it lacks the latter's extent of implementation and demonstrated utility. A psychometric approach alone is also incapable of effectively informing adaptation software, but it is potentially useful in combination with other methodology, primarily consisting of a production-system expert system (Buchanan \& Shortliffe, 1984; Clancey, 1985; Lachman, 1989a). Consequently, the strategy that has been adopted in the present research is a combination of keystroke analysis, psychometrics, and a production system expert.

The Carnegie Group (Hayes, 1988) recommends that an independent knowledge base be used to drive the interface for expert systems. The rationale for this recom- 
SPACE STATION FREEDOM

MPAC - DMS WORD PROCESSING SERVICES -- HELP SCREEN

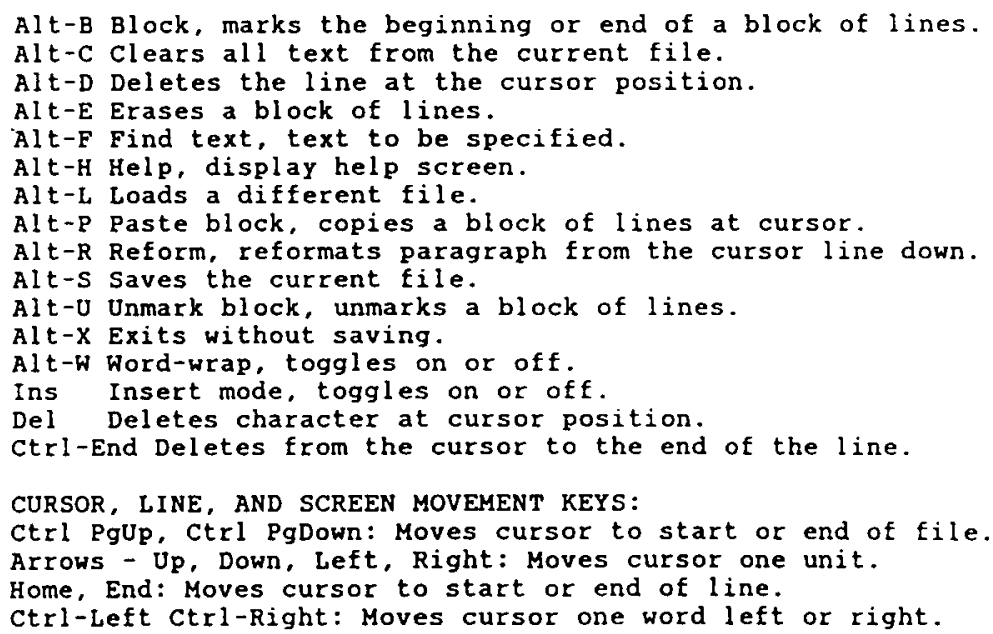

Figure 1. The help screen for a novice user, showing a limited command set for the word processor.

mendation includes ease of rule modification and consistency between the interface and the internal system with respect to how problems are viewed. The rule-based expert system under development is described below; it drives a workstation user service. This experimental expert system is coupled to application software, but it is designed to be consistent with the Carnegie recommendations (Hayes, 1988). The rule-based system exercises partial control over command syntax and command execution, as well as over the content of screen displays and advisory messages, for one workstation application and its interface. The recommendations were not developed for an integrated workstation, and many unresolved issues remain. For example, should the expert systems for various workstation services be integrated with a common knowledge base or be developed and maintained as separate units?

Published articles about research on the concept of an adaptive interface began to appear in 1980 . Yet no one has apparently reported a fully functional system, perhaps because of the prohibitive cost associated with a full-scale knowledge-engineering effort or other insurmountable problems. The current approach is cost-effective and perhaps conceptually feasible, because it starts with a scaled-down but fully functional software application and proceeds incrementally. In addition, the use of high-level domain experts is not required for prototype development. The workstation service selected for prototyping with a knowledge base, word processing, is a very common task with an established literature (Carroll, 1987). That literature, along with common information about the general properties of text editors, is the knowledge source for the construction of a rule base. Information in the literature is supplemented by direct observation of subjects performing the targeted task at a console. This effort is nonetheless experimental, and success is not guaranteed for reasons that I will describe later.

Once a tentative rule base is established, the adaptive system requires facts concerning the service in use, the state of the system, and the current performance of the user. Relevant facts are stored in the database of the expert system and are continuously compared to the antecedents of rules. When a match is obtained between the user's command entry and a rule, that rule "fires." The firing of a rule either adds a new fact to the database or issues a command to the underlying application software. Strictly speaking, adaptation produced by the expert system changes more of the application software than its interface. The firing of a set of rules designed to affect the application triggers commands that change the display configuration of the interface or the command structure of the underlying software system, or both. The user is then presented with automatic assistance or advice. The facts that are matched to the antecedents of rules are obtained from several sources, which include nonintrusive and embedded speed and accuracy measures of keystrokes or mouse movements at the workstation console; ruleselected automatic queries to the operator; unsolicited operator inputs and requests for help within predefined parameters; and software monitoring of the particular state of the workstation service being used.

Basically three types of procedures can be invoked by an adaptive interface system when all of the antecedents of a chain of rules are satisfied. First, the system can window a message onto the display screen, thereby telling the operator what keys to press in order to solve a 
problem, increase functionality, raise productivity, reduce errors, or recover from an error state. Second, the system can directly initiate the execution of one or more commands. Third, the system can query the user as to his or her intentions or initiate a dialogue to define and solve a problem. An example of the rules governing the first type of procedure (advisory windows) is shown below, and the action it produces is shown in Figure 2. The advisory message is windowed over the visible screen of the word processor by a rule with the following form:

RULE 14

IF LEARNING.LEVEL $<3$

AND IF RESPONSE(last) $=$ RIGHT.ARROW.KEY

AND IF RESPONSE (last -1$)=$ RIGHT.ARROW.KEY

AND IF RESPONSE (last -2 ) $=$ RIGHT.ARROW.KEY

AND IF RESPONSE(last -3 ) = RIGHT.ARROW.KEY

AND IF RESPONSE $($ last -4$)=$ RIGHT.ARROW.KEY

AND IF RESPONSE(last -5 ) $=$ RIGHT .ARROW.KEY

AND IF LINE.LENGTH(CURRENT.LINE) - CURSOR.POSITION $>9$

THEN PRINT RIGHT.WORD ;execute print command

The rule states that if the operator is a novice at Learning Level 2 or less, if the right-arrow key was pushed during each of the last six successive keystrokes, and if the cursor is now 10 or more character positions from the last character on the line, then print the window message shown in Figure 2.

The second type of procedure produced by the expert system executes a command in the application code instead of sending a message to the operator. One example of a command execution rule follows:
RULE 15

IF LEARNING.LEVEL $<3$

AND IF RESPONSE(last) $=$ CNTL.RIGHT .ARROW

AND IF RESPONSE(last -1$)=$ CNTL.RIGHT.ARROW

AND IF RESPONSE (last -2$)=$ CNTL.RIGHT.ARROW

AND IF RESPONSE (last -3$)=$ CNTL.RIGHT.ARROW

AND IF RESPONSE(last -4$)=$ CNTL.RIGHT.ARROW

AND IF LINE.LENGTH(CURRENT.LINE) - CURSOR.

POSITION $>5$

AND IF LINE.LENGTH(CURRENT.LINE) - CURSOR. POSITION $<16$

THEN END.KEY ;execute command end.key

The rule states that if the user is at Learning Level 1 or 2 , and if the last five keystrokes moved the cursor five words to the right and there are at least 6 characters left on the line but not more than 16, then execute the command to jump the cursor to the end of the line. The assumption is that the operator wanted to get to or near the end of the line and did not know the name of the command or forgot it. Obviously, empirical research on cognitive processes is necessary to determine which assumptions concerning operator intentions are viable and under what conditions; some experienced computer users appear to be disconcerted when the machine "takes over." This observation is based on a small sample and may be an isolated effect. If it turns out to be a more general response, then remedial action may be required-such as an advisory message explaining what was done and tutoring the operator on the appropriate keyboard input for the command. In addition, the operator can be given the option of disabling some or all automatic command execu-

3.0 GULDLINBg POR IUTRRACTIOMS BETWBEN USBRS MHD THB gSPP COMPUTBR 9Y9TEMS

The interactions between the varlous users and the sSpp coaputer systers can be characterized by three broad categories - the presentation of information to the user, real-time interactions between the user and the systen, and the user's the user, real-time interactions between the user and the systen, and the user

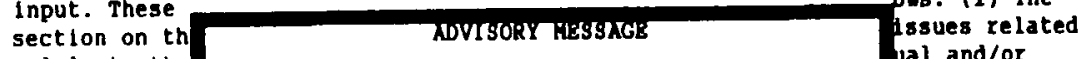
solely to the auditory moda If you want to move the cursor to the right ore processing of the displayed quickly, hold down Ctrl and press the Right of guidelines is concerned Arrov key, the cursor will junp to the right one display, the organization word at a time. the user's at Press 〈ANY KBY> TO CONT INUB. ding the meaning of dilon on real-tine Interactions between users and the ssep computer systems covers human-computer interfaces that involve a close conceptual and temporal relatio human-computer interaction cannot be easily classified as involving primarily elther inforation processing or response output. Several of the topics covered under this category previously have been labeled "direct manipulation interfaces". The real-time interactions in this category include sethods for moving between displays, windowing techniques, selecting information from a display, user guidance, and interactive dialogue techniques. (3) Input fron the LINE: 21 CHAR: 45 DMS Bditing File: GUIDELIN. Alt-H for Help.

Figure 2. An advisory message that is windowed onto the screen by Rule 14. The rule is described in the text. 
tion initiated by the adaptive interface. Obviously, command execution rules as well as rules that alter the screen display and command entry features require behavioral research to determine their effects on user satisfaction and productivity. This effort will have to be totally bottomup; there is no other way.

An important part of the task of the knowledge-based system is to determine the intentions of the human operator entering commands for a particular workstation service. One way to predict user intentions is to observe an expert trying to determine a user's intentions at a console and to capture the rules used by the expert when the expert is successful. Obviously, even a domain expert cannot perfectly determine the intentions of other people performing in the domain; this can only be done partially. A machine can do no better than the expert whose rules are being implemented. But people can partially predict the intentions of others and provide appropriate directions and advice; an expert-system machine implementation might be designed to do as well.

The most important reason for linking the expert-system prototype to word processing is the availability of source code. An adaptive interface can only be constructed for a software system if the source code is available. The code is either written or obtained from the copyright holder of a commercial system. Several types of word processors are in the public domain, and the literature contains numerous algorithms for text-editing functions such as reformat, find, or insert. The word processor was written thus for this project. At present, it is unclear whether or not word processing is too simple a task to require an adaptive interface for experienced users, even if they are occasional users. Even if word processing is an appropriate task, this does not mean that large-scale deployment is unproblematic. In either case, intensive and welldesigned behavioral experimentation is essential. The sampling of subjects must be done meticulously for realistic generalization to specialized and global populations. Scaling up to highly complex commercial systems and integrating a very large rule base may present significant technical problems. But these can be solved more readily than those dealing with human cognition.

In the first half of this article, I described the needs, objectives, and design philosophy for an adaptive interface. The second half contained an account of the properties of an operational prototype. The architecture of the adaptive interface is similar to the three-component architecture of a conventional expert system. First, there is a database with the operator's psychometric profile and prior performance history, as well as the output of the current keystroke performance stack. Second, there is a rule base with performance and error detection rules or productions in the form of conditionals between the operator's behavior and three types of instructions to the underlying application software. Third, there is an inference engine that searches the database for antecedents to rules and sends the consequents of fired rules as commands to the underlying software. This structure appears sufficient for the construction of adaptive capacities in workstation applications. Other methodologies such as artificial neural nets, machine learning (Fayyad, Laird, \& Irani, 1989; Kodratoff, 1988), and machine induction (Langley, Simon, Bradshaw, \& Zytkow, 1987) might extend the methods described in this paper. Technological support via machine learning is not likely to be available immediately, however. Although important properties of artificial neural networks have been studied, large-scale technological applications have yet to be reported.

\section{REFERENCES}

ANDERSON, J. R., FARRELl, R., \& SAUERS, R (1984). Leaming to program in LISP. Cognitive Science, 8, 87-129.

Buchanan, B. G., Shortliffe, E. H. (Eds.). (1984) Rule-based expert systems. Reading, MA: Addison-Wesley.

Card, S. K., Moran, T. P , Newell, A. (1983). The psychology of human-computer interaction. Hillsdale, NJ: Erlbaum.

CARroll, J. M. (Ed.). (1987). Interfacing thought: Cognitive aspects of human computer interaction. Cambridge, MA: MIT Press.

Chi, M. T. H., Glaser, R , ReEse, E. (1982). Expertise in problem solving. In R. Sternberg (Ed.), Advances in the psychology of human intelligence (pp. 7-75). Hillsdale, NJ: Erlbaum.

Clancey, W. J. (1985). Heuristic classification. Artificial Intelligence, 27, 1-67.

DAvis, R (Ed.). (1989). Expert systems: How far can they go?-Part two. Al Magazine, 10, 65-77.

FAYYAD, U. M., Laird, J E., IRANI, K. B (1989). The fifth international conference on machine learning. Al Magazine, 10,79-84

HAYEs, P. J. (1988). Using a knowledge base to drive an exper system interface with a natural language component. In J. A. Hendler (Ed.), Expert systems The user interface (pp. 153-182). Norwood, NJ: Ablex

Hoppe, (1988) Task-oriented parsing-A diagnostic method to be used by adaptive systems. In Proceedings of CHI 88 (pp. 241-247) New York: Association for Computing Machinery.

KoDra toff, Y. (1988). Introduction to machine learning. San Mateo, CA. Morgan Kaufmann.

LaChman, R. (1989a) Expert systems: A cognitive science perspective. Behavior Research Methods, Instruments, \& Computers, 21, 195-204.

LACHMAN, R. (1989b). On-line reading and comprehension aids for expository text. Human Factors, 31, 1-15

Langley, P., Simon, H. A., Bradshaw, G. L., \& Zytkow, J. M. (1987). Scientific Discovery. Cambridge, MA: MIT Press.

Luchier, R. E., Matheson, N. W., Butter, K. A., \& Reynolds, R. E. (1988). The knowledge workstation: An electronic environment for knowledge management. Bulletin of the Medical Library Association, 76, 248-255.

National Aeronautics and Space Administration. (1989). Preliminary draft: Architectural control document, data management system (SSP 30261, Draft Version 2, Revision D). Space Station Freedom Program Office.

Payne, S. J , G Green, T. R. G. (1986). Task action grammars-A model of the mental representation of task languages. Human-Computer Interaction, 2, 93-133.

Polson, P. G., KIERAS, D (1985). A quantitative model of the learning and performance of text editing knowledge. In Proceedings of $\mathrm{CHI}$ 85 (pp. 207-212). New York. Association for Computing Machinery.

Rouse, W. B., Geddes, N. D., Curry, R E. (1987-88). An atchitecture for intelligent interfaces: Outline of an approach to supporting operators of complex systems. Human-Computer Interaction, 3, 87-122.

TYLER, S. W. (1988). SAUCI: A knowledge-based interface architecture. In Proceedings of CHI 88 (pp. 235-240). New York. Association for Computing Machinery. 